\title{
Multitrack electrospray chips
}

\section{Loïc Dayon, ${ }^{\dagger}$ Mélanie Abonnenc, ${ }^{\dagger}$ Michel Prudent, ${ }^{\dagger}$ Niels Lion and Hubert H. Girault*}

Laboratoire d'Electrochimie Physique et Analytique, Ecole Polytechnique Fédérale de Lausanne (EPFL), Station 6, CH-1015 Lausanne, Switzerland

Received 9 August 2006; Accepted 6 September 2006

\begin{abstract}
Multitrack electrospray chips (MTEC) were fabricated by UV-photoablation of polyethylene terephthalate (PET) substrates. They are composed of an array of up to six microchannels that are successively used as electrospray ionization (ESI) emitters for mass spectrometry (MS). There is no requirement for alignment of the different spraying microchannels with the mass spectrometer orifice. The MTEC is thus fixed in front of the mass spectrometer and the successive MS analyses are performed without moving the chip. The sequential electrospraying by successive application of an identical high voltage in each off-axis microchannel was evaluated for the fast screening of peptides and proteins. The counting of cysteines in peptides through chemical modification and the relative quantification of a peptide in two samples are presented herein as two original strategies based on this new analytical tool. Copyright @ 2006 John Wiley \& Sons, Ltd.
\end{abstract}

KEYWORDS: chip; electrospray; emitter; mass spectrometry; quantification; sequential; source; tagging

\section{INTRODUCTION}

Since the 1990s, microtechnologies have offered many options to fabricate devices for applications in the field of analytical sciences and especially in mass spectrometry (MS).

Electrospray ionization (ESI) MS is a method of choice for the determination of the mass of biomolecules. By applying a high electric field between a capillary tip and the mass spectrometer, a spray of highly charged droplets is produced at the extremity of the tip. The charged droplets reduce in size by evaporation of the solvent and/or by Coulomb explosion to form gas-phase ions representative of the species in solution. ${ }^{1-4}$ These ions are then analyzed and detected by the mass spectrometer.

Microfabrication allows a large panel of choices for the design of the ESI emitter in terms of material (glass, silica, polydimethylsiloxane, polyethylene terephthalate (PET)), size and shape of the structures. ${ }^{5}$ Micro- and nanospray have been widely studied. They were demonstrated to provide better sensitivity than the standard ESI sources commonly used in MS. The advantages of miniaturized ESI microchip emitters are multiple. First, microfabrication enables the incorporation of several ESI emitters on the same chip, which is effective for parallel and high-throughput analyses. Second, on-chip functionalization such as sample preparation and/or separation can be hyphenated with the ESI emitter.

Several groups introduced multispray emitter chips some years ago. In particular, parallel microchannels with

*Correspondence to: Hubert H. Girault, Laboratoire d'Electrochimie Physique et Analytique, Ecole Polytechnique Fédérale de Lausanne (EPFL), Station 6, CH-1015 Lausanne, Switzerland. E-mail: hubert.girault@epfl.ch

${ }^{\dagger}$ Equal Authorship. reservoirs ${ }^{6}$ or ESI emitter arrays $^{7}$ were positioned on a computer-controlled translation stage for serial analyses of samples. The emitters were successively aligned in front of the mass spectrometer orifice. Henion's group and Advion Biosciences developed silicon ESI chips with an array of nozzles that were used for high-throughput MS quantification of drugs from plasma samples. ${ }^{8,9}$ A dual sprayer ion source was also reported for the accurate mass measurement of compounds by time-of-flight (TOF) or Fourier transform ion cyclotron resonance (FTICR) MS when internal calibration was required. ${ }^{10-13}$ The two electrospray emitters were positioned orthogonally to each other or in parallel on a rotating platform in front of the mass spectrometer entrance. One sprayer was used to ionize the analyte while the other sprayer provided the calibrant. By switching the voltage between the emitters, the analyte and calibrant were analyzed sequentially. Such dual sprayers often require a complex apparatus and, in most cases, a motorized stage to place the successive emitters on the axis of the mass spectrometer orifice. Other applications of the multi-ESI emitters are the studies of ion/ion interaction by switching the ESI between positive and negative ionization modes, ${ }^{14}$ mixing and reaction in the Taylor's cone. ${ }^{15,16}$

In the present study, a multitrack electrospray chip (MTEC) was designed. The microchannels were patterned by UV-photoablation of PET sheets. ${ }^{17,18}$ The uniqueness of the device is the incorporation of up to six ESI emitters on a single microchip. The microfabrication protocol is fast and easily flexible as regards the dimensions of the microchannels (typical section of $100 \times 40 \mu \mathrm{m}^{2}$ and length of $1.5 \mathrm{~cm}$ ) and the distance between them (4-8 $\mathrm{mm}$ ). The second key feature of the MTEC setup is the fixed positioning of the microchip in front of the mass spectrometer. The samples are therefore electrosprayed off the axis of the mass spectrometer orifice. 
Such a microchip is effective for fast screening applications in analytical chemistry and proteomics. Several proteins and peptides were alternatively electrosprayed from each track by switching the voltage between the microchannels. In addition, a two-track microchip was used for the counting of cysteines in peptides. The serial two-track ESI-MS analysis allows the obtaining of the mass of the untagged peptides (reference) and that of the cysteine-derivatized peptides in a single experiment. The relative quantification of a peptide between two samples was also carried out using the twotrack version of the MTEC.

\section{EXPERIMENTAL}

\section{Chemicals}

Angiotensin III (RVYIHPF, >97\%, M $=931.1 \mathrm{~g} \mathrm{~mol}^{-1}$ ), angiotensin I triacetate salt (DRVYIHPFHL, $>99 \%, M=$ $1269.5 \mathrm{~g} \mathrm{~mol}^{-1}$ ), Leu-enkephalin (YGGFL, >99\%, $M=$ $\left.555.6 \mathrm{~g} \mathrm{~mol}^{-1}\right)$ and KCTCCA $\left(70 \%, M=627.8 \mathrm{~g} \mathrm{~mol}^{-1}\right)$ were bought from Bachem (Bubendorf, Switzerland). Bradykinin (RPPGFSPFR, 99\%, $M=1060.2 \mathrm{~g} \mathrm{~mol}^{-1}$ ) and myoglobin from horse heart $(90 \%)$ were from Sigma (St Louis, MO, USA) and cytochrome $c$ from horse heart ( $\geq 95 \%), 1,4-$ benzoquinone (BQ) $\left(\geq 98 \%, M=108.1 \mathrm{~g} \mathrm{~mol}^{-1}\right)$ and acetic acid (>99.5\%) were from Fluka (Büchs, Switzerland). Keratin K18 (RPVSSAApSVYAGAC, >95\%, M $=1418.5 \mathrm{~g} \mathrm{~mol}^{-1}$ ) was from AnaSpec (San José, CA, USA). Methanol (>99.8\%) was from Riedel-de-Haën (Seelze, Germany). Water (UVHPLC) and acetonitrile (HPLC-gradient grade) were from Panreac Quimica S.A. (Barcelona, Spain). All the chemicals were used without any further purification.

The spray medium was $\mathrm{MeOH} / \mathrm{H}_{2} \mathrm{O} / \mathrm{AcOH}$ (50\%/49\%/ $1 \%)$. The concentrations of the peptides and proteins were $50 \mu \mathrm{M}$ and $5 \mu \mathrm{M}$, respectively. Cysteines in KCTCCA were derivatized by mixing the peptide at $50 \mu \mathrm{M}$ in the acidic spray medium with $5 \%(\mathrm{v} / \mathrm{v})$ of $\mathrm{BQ}$ at $100 \mathrm{~mm}$ in $\mathrm{CH}_{3} \mathrm{CN}^{19}$ The reaction was performed at room temperature for $60 \mathrm{~min}$. For quantification experiments, KCTCCA concentrations were 12.5, 25 and $50 \mu \mathrm{M}$. All solutions were prepared freshly every day.

\section{Microchip device}

The MTEC was microfabricated according to a simple procedure $^{18,20}$ A PET polymer sheet of $100 \mu \mathrm{m}$ thickness (Melinex sheet from Dupont, Wilmington, DE, USA) was ablated by the UV-light of an excimer laser (Lambda Physik, Göttingen, Germany). The polymer substrate was mounted on an $x-y$ table, perpendicularly to the laser beam. A rectangular mask was placed before the substrate to define the ablation area. The microchannel pattern (Fig. 1) was drilled by translation of the $x-y$ table, moving the sample during irradiation. The depth of the microchannel was adjusted by setting the speed of the $x-y$ table. At the ends of the microchannel, reservoirs were made by static laser shots which drilled the PET sheet right through. The MTEC device was obtained by drilling on a single PET sheet several parallel microchannels at distances $d$ from each other. The microchannel shape was inspected and the dimensions were controlled using a microscope, which enabled the measurement of the length, width and depth of the structure. After laser ablation, the chip was cleaned with $\mathrm{MeOH}$ or isopropanol and dried under nitrogen. The PET substrate was then laminated to close the structures. In this way microchannels with two reservoirs at their extremities were obtained. In the lamination process, a thin PET foil coated with a melting adhesive layer of polyethylene (PE) (PET of $25 \mu \mathrm{m}$ thickness with a $10 \mu \mathrm{m}$ thick adhesive PE, from Morane, Oxon, UK) was rolled onto the PET sheet with a heated roller $\left(130^{\circ} \mathrm{C}\right.$ and 3 bars for $3 \mathrm{~s})$. The chip was placed at $80^{\circ} \mathrm{C}$ for $45-60 \mathrm{~min}$ between two glass plates to allow adhesion of the lamination layer to the structure. The final V-shaped nozzles were obtained by cutting the microchip (i.e. one extremity of the microchannel) with a pair of scissors. The microchannel section was $100 \times 40 \mu \mathrm{m}^{2}$ and the distance $d$ between successive parallel microchannels was 4 or $8 \mathrm{~mm}$. The microchannel length was $1-1.5 \mathrm{~cm}$. A polycarbonate well (diameter $\phi=6.5 \mathrm{~mm}$ ) was fixed at the inlet (i.e. reservoir) of each microchannel (Fig. 1).

\section{Mass spectrometry}

A LCQ DUO ion trap mass spectrometer (Thermo Electron, San José, USA) was used for ESI-MS. The heated capillary was kept at $200^{\circ} \mathrm{C}$. The ESI interface was removed and the MTEC was mounted on a plate fixed on the probe slide adapter of the mass spectrometer. The MTEC was centered in front of the mass spectrometer orifice (Fig. 1). For a chip
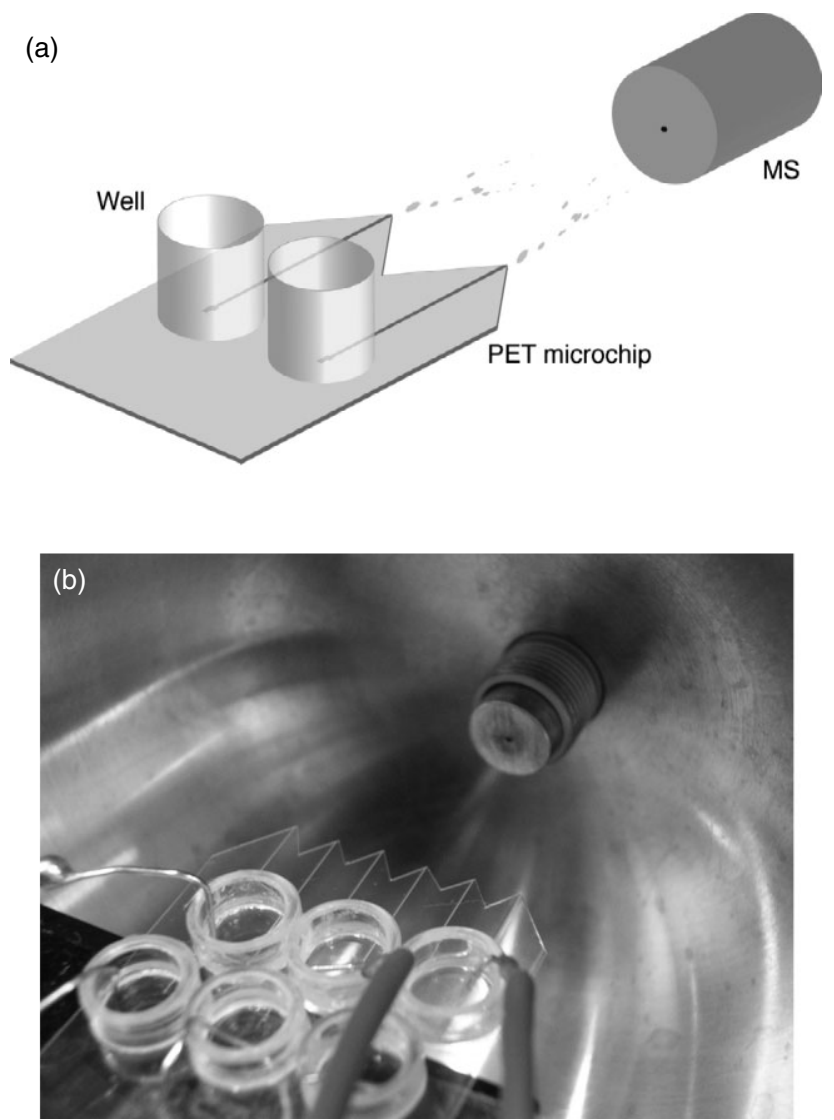

Figure 1. Multitrack electrospray chip (MTEC): (a) schematic representation of a two-track chip; (b) picture of an MTEC comprising 6 microchannels. 
with an even number of tracks, the microchannels are thus all positioned parallel but off the axis of the mass spectrometer orifice. Samples were loaded (sample volume $V=50 \mu \mathrm{l}$ ) in the wells, providing a typical flow rate of $600 \mathrm{nl} \mathrm{min}{ }^{-1} \cdot{ }^{21} \mathrm{In}$ each well, a platinum wire electrode was inserted. A high potential $(4 \mathrm{kV})$ was applied to the sample using the internal voltage of the mass spectrometer. It was switched manually to the next sample using an external source of voltage $(4 \mathrm{kV})$ at time intervals $\Delta t=30,15$ or $6 \mathrm{~s}$. After another $\Delta t$, the next serial electrospraying was achieved using the mass spectrometer internal voltage again, etc. The distance from the outlet of the microchannel to the entrance of the mass spectrometer was varied between 1.5 and $2 \mathrm{~cm}$ in order to optimize the signal and the trap injection time. Optimization of mass spectrometer parameters was realized on the analyte of interest. Each experiment was repeated and reproduced with different chips.

\section{RESULTS}

The MTEC was first evaluated in the simple case of a chip comprising two parallel microchannels at a distance $d=8 \mathrm{~mm}$, which was appropriate to prevent solution mixing (Fig. 1(a)). Peptide samples were sequentially electrosprayed. Angiotensin III and bradykinin (or Leu-enkephalin) were placed in the different reservoirs of the microchannels. The voltage provided by the MS was applied to the first sample (angiotensin III) for $30 \mathrm{~s}$. While the voltage of the MS was switched to $0 \mathrm{~V}$, an identical voltage was applied with an external source to the second sample (bradykinin or Leuenkephalin) for $30 \mathrm{~s}$. The switching sequence was repeated 10 times (data not shown). The stability and reproducibility of the experiments were demonstrated. The chips were reused after washing with a spray medium solution. Protein samples of myoglobin and cytochrome $c$ were successively electrosprayed as described above. The mass spectra were recorded on half a sequence $(\Delta t=30 \mathrm{~s})$, one sequence $(2 \Delta t=60 \mathrm{~s})$

(a)

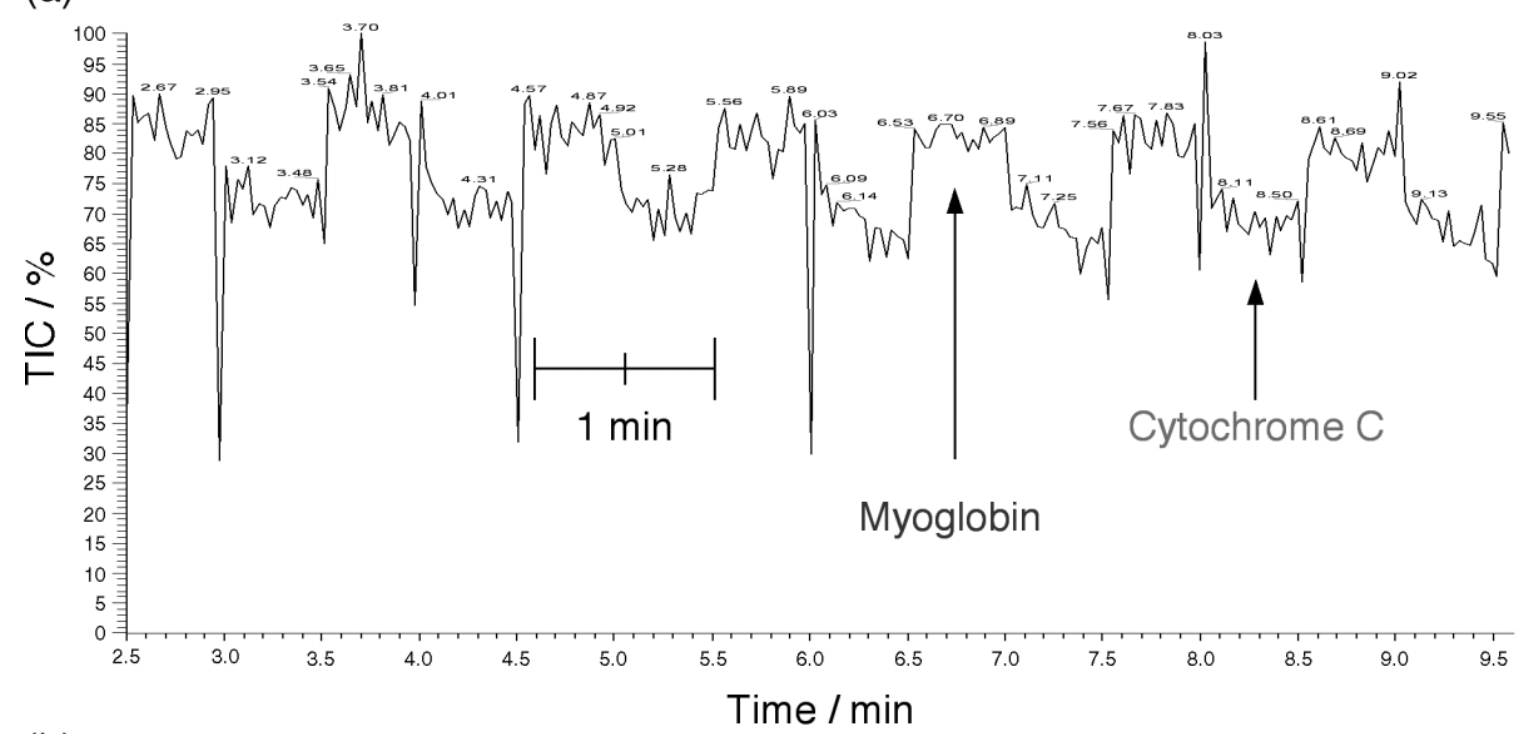

(b)

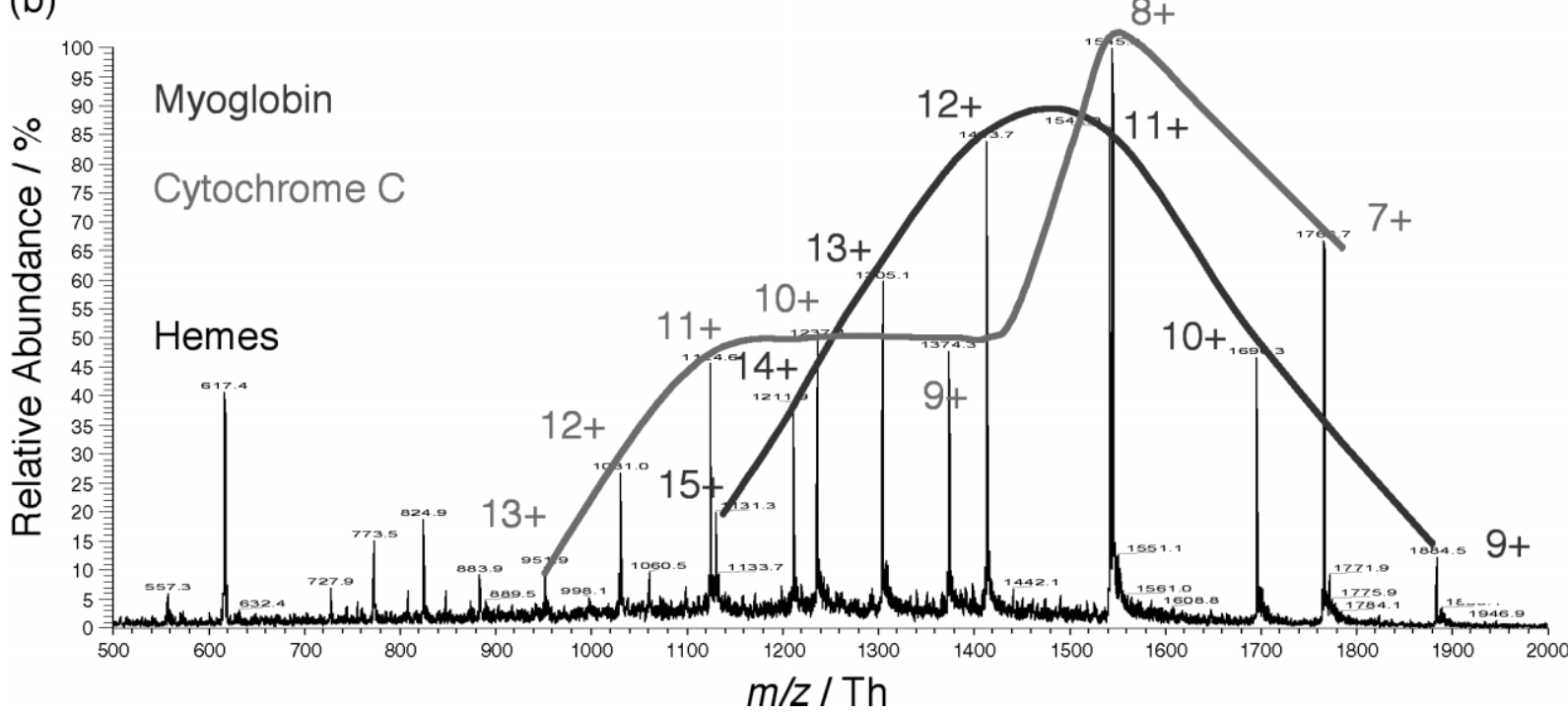

Figure 2. (a) Total ion current (TIC) and (b) cumulative mass spectrum of myoglobin and cytochrome $c$. The average cumulative mass spectrum was obtained by integration of the scans for $8 \mathrm{~min}$. Each switch was done after $\Delta t=30 \mathrm{~s}$. The TIC signal clearly shows the difference in ionization efficiency of the two proteins. 
or several sequences of voltage switch in order to split or gather the MS data from each sample (Fig. 2). The total ion current (TIC) was monitored. It is representative of the voltage switch and shows the difference in the sample ionization efficiencies. Myoglobin was shown to ionize better than cytochrome $c$ during the ESI-MS analysis.

An MTEC emitter with six microchannels was microfabricated (Fig. 1(b)) and tested with peptide samples. No mixing of the sample solutions was detected although $d$ was decreased to $4 \mathrm{~mm}$. Solutions of KCTCCA, angiotensin III, Leu-enkephalin, angiotensin I, keratin K18 and bradykinin were placed separately in the six microchannel reservoirs and sequentially electrosprayed by successive application of the voltage (from the mass spectrometer or the external source). The TIC was recorded for $12 \mathrm{~min}$. The superposition of the mass spectra shown in Fig. 3(b) demonstrates that the MTEC device allows rapid screening of all six samples.

The MTEC was further used for the counting of cysteines in peptides. 1,4-Benzoquinone was employed as reagent to chemically label the cysteines of KCTCCA in the ESI medium. ${ }^{19}$ After complete derivatization, a two-track chip $(d=8 \mathrm{~mm})$ was positioned in front of the mass spectrometer (Fig. 1(a)). One reservoir was filled with the KCTCCA solution and the other with the labeled peptide at the same concentration $(50 \mu \mathrm{M})$. The solutions were electrosprayed successively (Fig. 4(a)). The collection of mass scans on a selected time window gives access to the peptide, the tagged peptide or both (Fig. 4(c), (d) and (b) respectively). When monitoring the whole sequence $(2 \Delta t)$, the number of cysteines in the peptide can be easily determined (Fig. 4(b)). From the difference of the mass of the peptide to that of the tagged peptide, the number of mass shifts due to the tag enables the counting of cysteine residues. ${ }^{22}$ In addition, the TIC signal clearly indicates that the quinone-tagged KCTCCA ionizes slightly better than KCTCCA (Fig. 4(a)). This tag-induced ionization effect has already been observed in the case of $\beta$-lactoglobulin A. ${ }^{23}$ The voltage switch interval $\Delta t$ was reduced from $30 \mathrm{~s}$ to $15 \mathrm{~s}$ and $6 \mathrm{~s}$ (Fig. 4(a)). When
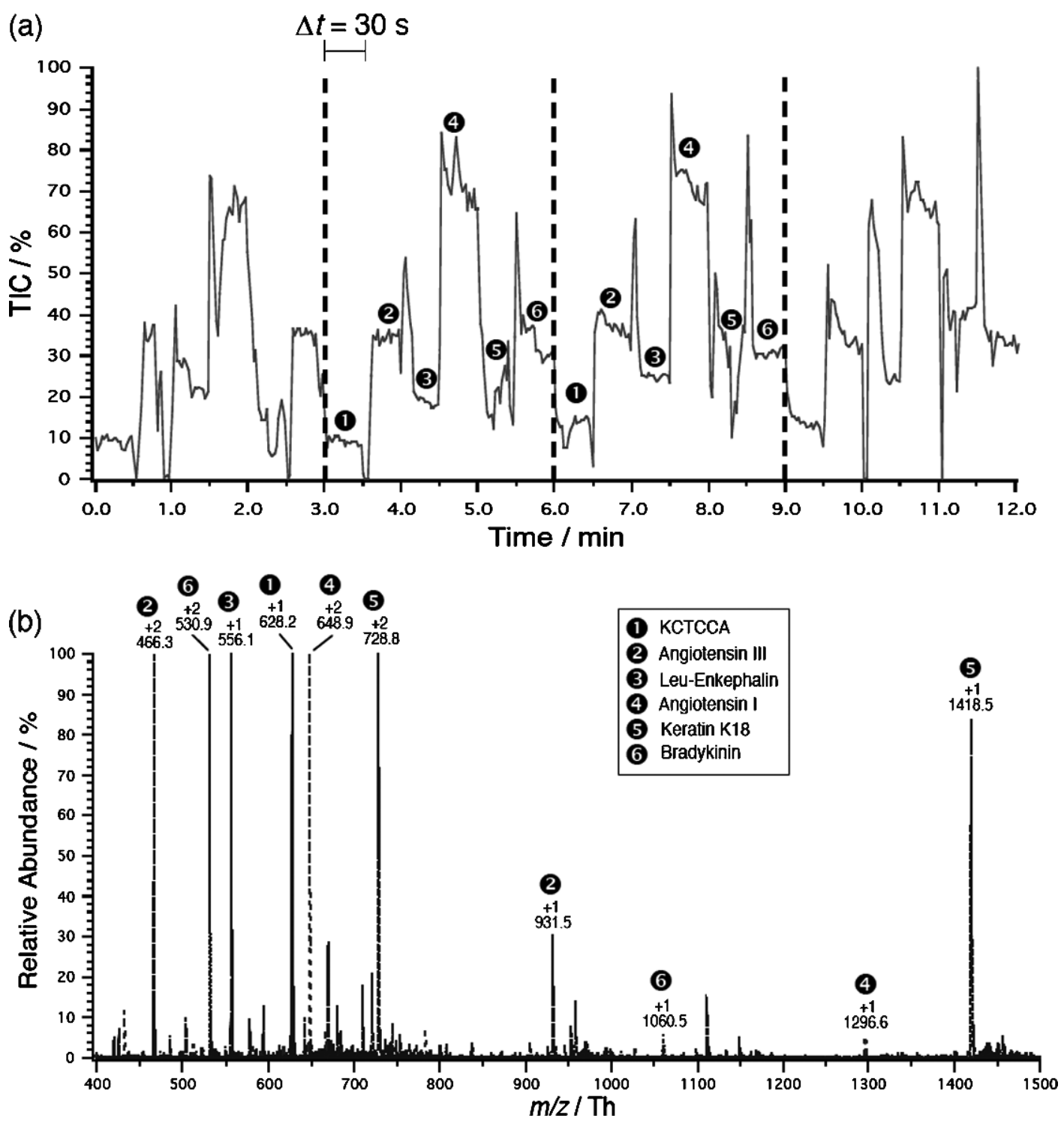

Figure 3. Screening of KCTCCA (1), angiotensin III (2), Leu-enkephalin (3), angiotensin I (4), keratin K18 (5) and bradykinin (6) performed successively with a six-track microchip. Each sample was analyzed for $30 \mathrm{~s}$. (a) Total ion current signal of 12 min corresponding to four screening sequence of the six samples. (b) Superposed mass spectra of the six compounds. 

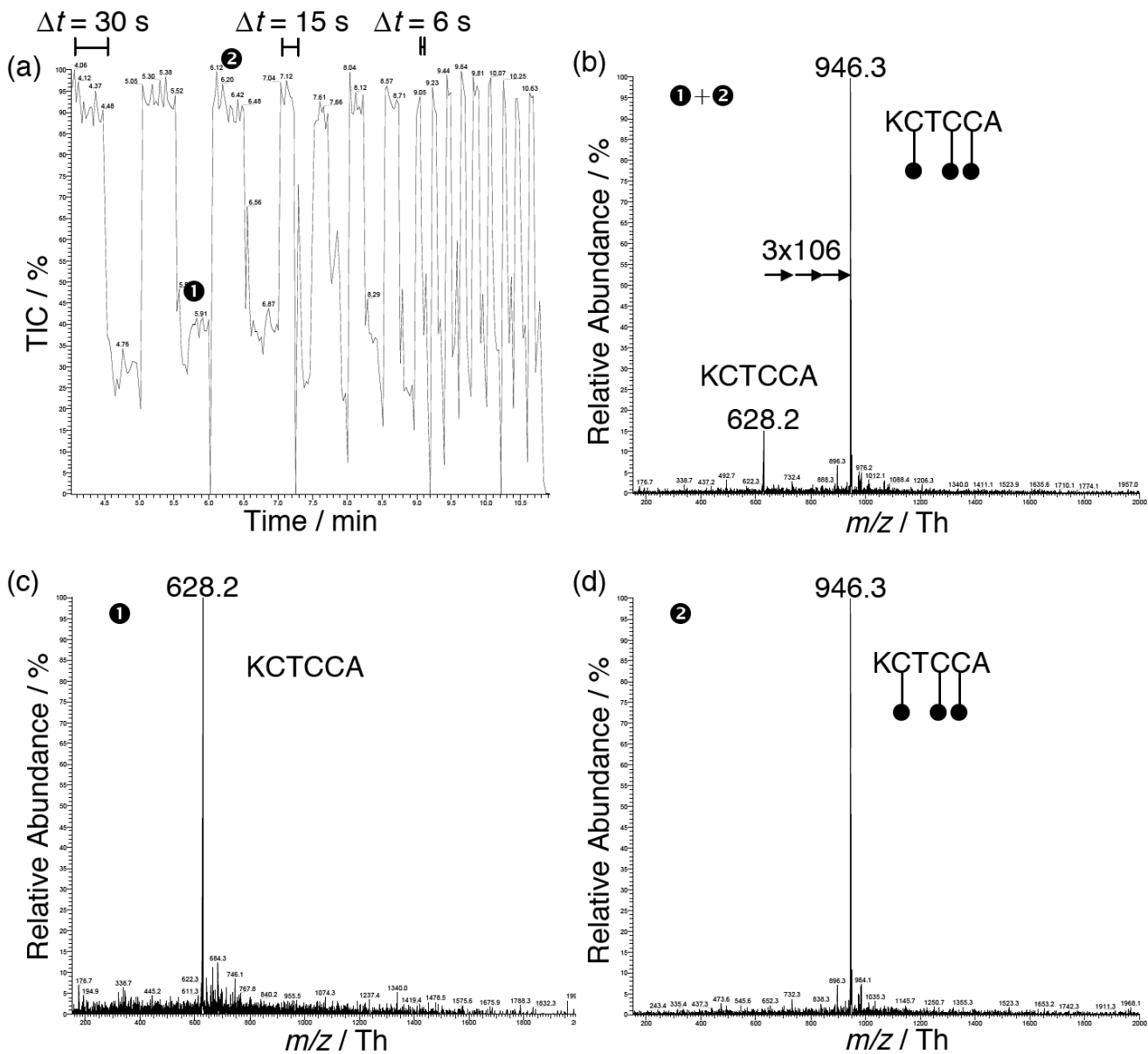

Figure 4. Two-track electrospraying of KCTCCA and its cysteinyl-tagged analog. (a) Total ion current signal with decreasing switch time interval $\Delta t$. (b) Mass spectrum of both species $(1+2)$ averaged over $12 \mathrm{~s}(\Delta t=6 \mathrm{~s})$. (c) Mass spectrum of KCTCCA (1) averaged over $6 \mathrm{~s}$. (d) Mass spectrum of cysteinyl-tagged KCTCCA (2) averaged over $6 \mathrm{~s}$.
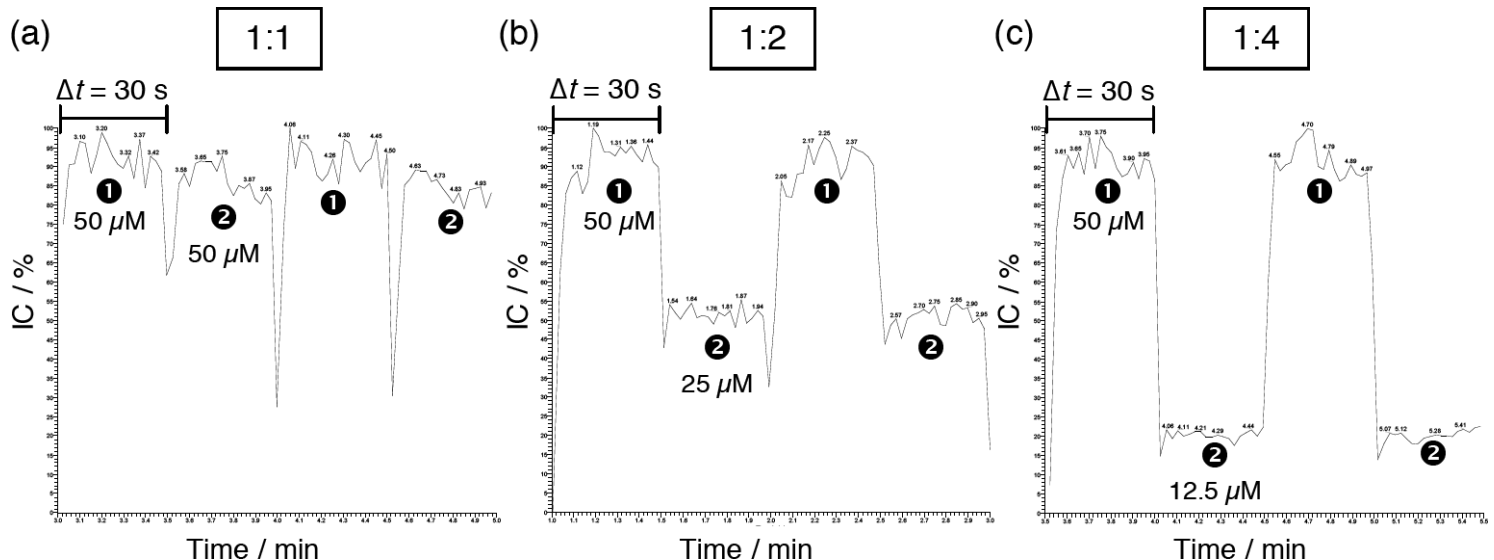

Figure 5. Relative quantification of KCTCCA in two samples using a two-track microchip. (a) lon current (IC) signal at $m / z=628.2$ Th for KCTCCA $50 \mu \mathrm{M}$ (2) and $50 \mu \mathrm{M}$ (1) (ratio of 1). (b) IC signal at $m / z=628.2$ Th for KCTCCA $25 \mu \mathrm{M}$ (2) and $50 \mu \mathrm{M}$ (1) (ratio of 0.5 ). (c) IC signal at $m / z=628.2$ Th for KCTCCA $12.5 \mu \mathrm{M}$ (2) and $50 \mu \mathrm{M}$ (1) (ratio of 0.25 ).

switching every $6 \mathrm{~s}$, the analysis of both samples was still perfectly achieved (Fig. 4(c)-(d)).

The two-track chip (Fig. 1(a)) was used to electrospray two KCTCCA samples at different concentrations. When using the two-track version of the MTEC, both the symmetric microchannels were located at equal distances from the axis of the mass spectrometer orifice, providing identical spraying conditions. Experiments with two samples of KCTCCA at $12.5 \mu \mathrm{M} / 50 \mu \mathrm{M}, 25 \mu \mathrm{M} / 50 \mu \mathrm{M}$ and $50 \mu \mathrm{M} / 50 \mu \mathrm{M}$ were carried out. The MS data provides the relative quantification of the samples (Fig. 5). The ion current (IC) relative to KCTCCA and its mass peak intensity shows the difference in the relative amount of the peptide in the sample. For $12.5 \mu \mathrm{M} / 50 \mu \mathrm{M}$ (theoretical ratio of 0.25 ) the averaged mass peak intensity 
ratio of the two samples is 0.22 (Fig. 5(c)), while it is 0.52 for $25 \mu \mathrm{M} / 50 \mu \mathrm{M}$ (theoretical ratio of 0.5 ) (Fig. 5(b)). The control experiment with KCTCCA at $50 \mu \mathrm{M} / 50 \mu \mathrm{M}$ confirms an equivalent ESI efficiency in both the microchannels (Fig. 5(a)); the averaged peak intensity ratio of the two identical samples is 0.94 (theoretical ratio of 1 ). These data were confirmed by a series of measurements with angiotensin III (data not shown).

\section{DISCUSSION}

A rapid screening of samples was performed using the MTEC device. This multimicrochannel chip was used without alignment of the microchannel nozzles with the orifice of the mass spectrometer. Therefore, there is no requirement for a 3D stage during the experiment. The efficiency of the ESI was shown to vary according to the position of the microchannel from the axis of the MS orifice (data not shown). The MS analysis (i.e. the electrospraying of the solution) was achieved up to $15 \mathrm{~mm}$ from the axis. This study demonstrates that the MTEC can be used to introduce an internal mass calibrant. In TOF- or FTICR-MS, the MTEC setup can thus be advantageously employed to achieve high mass measurement accuracy.

Moreover, the MTEC device serves for the counting of specific amino acid residues. The counting of cysteines in a peptide was demonstrated. Although the technique requires a separation of the initial sample, the information provided by the knowledge of the content of a rare amino acid such as cysteine was shown to improve protein identification by constraining the database search during peptide mass fingerprinting. ${ }^{22,24,25}$ Using the MTEC, the sample is rapidly (in a few seconds) compared to its tagged analogs by switching the voltage. By comparison of the two mass signals, the amino acid content is assessed through the detected mass shift. The counting strategy may therefore be applied to the counting of many other amino acids when quantitatively and specifically modified by a mass tag. ${ }^{19,26,27}$ By extension to synthetic chemistry, these data highlight the potential of the MTEC for the high-throughput screening of reactions in solutions.

Another remarkable result obtained with the MTEC concerns the direct comparison of the ionization efficiency of molecules and the relative quantification of samples. When a symmetric chip is used (i.e. when sample solutions are electrosprayed at symmetric positions from the axis of the MS orifice), the relative ionization efficiency of two samples can be probed. All results clearly show that the IC signal varies according to the sample. Besides, the separation of the flow stream of the analytes prevents preferential ionization and subsequent signal suppression of certain molecules as well as difference in concentrations and various electrospraying conditions. ${ }^{28}$ When two samples ionize similarly (e.g. the same peptide but at a different concentration), the relative quantification is directly achieved by comparison of the IC or the mass peak intensities of the two samples on the averaged mass spectra. The relative quantification of KCTCCA samples was clearly demonstrated. These results are very promising for the development of micro total analysis system ( $\mu$ TAS) for the evaluation of up- and downregulation of proteins. ${ }^{29}$

Lastly, the voltage switch was reduced to $6 \mathrm{~s}$, which still allowed a valuable analysis of the samples. The decrease of the analysis time offers an enhancement of the dynamic potential of the MTEC. However, the switching time interval remains too large at present for the accumulation of two ion populations in the trap of a mass spectrometer. Nepomuceno et al. accomplished accumulation of the analyte and the internal calibrant by decreasing the switching time to $50 \mathrm{~ms}{ }^{11}$

\section{CONCLUSIONS}

MTEC consists of an array of parallel microchannels that operate sequentially as ESI emitters. Its microfabrication is relatively inexpensive and fast. The drilling/design of a MTEC with six microchannels takes $20 \mathrm{~min}$. The lamination and the reheating are batch-processed ( $\sim 60 \mathrm{~min})$. The device is highly reproducible and robust. The advantage of such a technology is that it allows the screening of samples in a very short time. As ESI can occur off the axis of the mass spectrometer, there is no requirement to move the MTEC in front of the apparatus when switching the voltage (i.e. the sample). Moreover, the device is set to offer multiple applications: internal mass calibration, reaction evaluation (e.g. the counting of cysteine residues in biomolecules), ionization efficiency probing and relative quantification of an analyte.

Future developments of the MTEC will include the embedding of electrodes in the microchannels and operation under flow conditions ${ }^{20}$ to couple the device with liquid chromatography. The decrease of the voltage switching time will be assessed, opening the way to the study of gas-phase chemistry.

\section{Acknowledgements}

The Fond National Suisse pour la Recherche Scientifique is thanked for financial support.

\section{REFERENCES}

1. Dole M, Mack LL, Hines RL. Molecular beams of macroions. J. Chem. Phys. 1968; 49: 2240.

2. Iribarne JV, Thomson BA. Evaporation of small ions from charged droplets. J. Chem. Phys. 1976; 64: 2287.

3. Fenn JB, Mann M, Meng CK, Wong SF, Whitehouse CM. Electrospray ionization for mass spectrometry of large biomolecules. Science 1989; 246: 64.

4. Cole RB. Electrospray Ionization Mass Spectrometry (1st edn). John Wiley and sons: New York, 1997.

5. Lion N, Rohner TC, Dayon L, Arnaud IL, Damoc E, Youhnovski N, Wu ZY, Roussel C, Josserand J, Jensen H, Rossier JS, Przybylski M, Girault HH. Microfluidic systems in proteomics. Electrophoresis 2003; 24: 3533.

6. Xue Q, Foret F, Dunayevskiy YM, Zavracky PM, McGruer NE, Karger BL. Multichannel microchip electrospray mass spectrometry. Anal. Chem. 1997; 69: 426.

7. Liu HH, Felten C, Xue QF, Zhang BL, Jedrzejewski P, Karger BL, Foret F. Development of multichannel devices with an array of electrospray tips far high-throughput mass spectrometry. Anal. Chem. 2000; 72: 3303.

8. Dethy JM, Ackermann BL, Delatour C, Henion JD, Schultz GA. Demonstration of direct bioanalysis of drugs in plasma using 
nanoelectrospray infusion from a silicon chip coupled with tandem mass spectrometry. Anal. Chem. 2003; 75: 805.

9. Schultz GA, Corso TN, Prosser SJ, Zhang S. A fully integrated monolithic microchip electrospray device for mass spectrometry. Anal. Chem. 2000; 72: 4058.

10. Satomi $Y$, Kudo $Y$, Sasaki K, Hase T, Takao T. Accurate mass measurement in nano-electrospray ionization mass spectrometry by alternate switching of high voltage between sample and reference sprayers. Rapid Commun. Mass Spectrom. 2005; 19: 540.

11. Nepomuceno AI, Muddiman DC, Bergen Iii HR, Craighead JR, Burke MJ, Caskey PE, Allan JA. Dual electrospray ionization source for confident generation of accurate mass tags using liquid chromatography Fourier transform ion cyclotron resonance mass spectrometry. Anal. Chem. 2003; 75: 3411.

12. Zhou F, Shui W, Lu Y, Yang P, Guo Y. High accuracy mass measurement of peptides with internal calibration using a dual electrospray ionization sprayer system for protein identification. Rapid Commun. Mass Spectrom. 2002; 16: 505.

13. Wolff JC, Eckers C, Sage AB, Giles K, Bateman R. Accurate mass liquid chromatography /mass spectrometry on quadrupole orthogonal acceleration time-of-flight mass analyzers using switching between separate sample and reference sprays. 2 . Applications using the dual-electrospray ion source. Anal. Chem. 2001; 73: 2605

14. Xia Y, Liang XR, McLuckey SA. Pulsed dual electrospray ionization for ion/ion reactions. J. Am. Soc. Mass Spectrom. 2005; 16: 1750.

15. Lion N. Microfluidic front-end technologies for protein electrospray mass spectrometry. PhD Thesis, Ecole Polytechnique Fédérale de Lausanne, 2006.

16. Chen YR, Tseng MC, Her GR. Design and performance of a lowflow capillary electrophoresis-electrospray-mass spectrometry interface using an emitter with dual beveled edge. Electrophoresis 2005; 26: 1376

17. Srinivasan R, Maynebanton V. Self-developing photoetching of poly(ethylene-terephthalate) films by far ultraviolet excimer laser-radiation. Appl. Phys. Lett. 1982; 41: 576.
18. Roberts MA, Rossier JS, Bercier P, Girault H. UV laser machined polymer substrates for the development of microdiagnostic systems. Anal. Chem. 1997; 69: 2035.

19. Dayon L. Thiol-targeted Microspray Mass Spectrometry of Peptides and Proteins through On-line EC-tagging. PhD Thesis, Ecole Polytechnique Fédérale de Lausanne, 2006.

20. Rohner TC, Rossier JS, Girault HH. Polymer microspray with an integrated thick-film microelectrode. Anal. Chem. 2001; 73: 5353.

21. Morier P, Vollet $C$, Michel PE, Reymond F, Rossier JS. Gravity-induced convective flow in microfluidic systems: electrochemical characterization and application to enzymelinked immunosorbent assay tests. Electrophoresis 2004; 25: 3761.

22. Dayon L, Roussel C, Prudent M, Lion N, Girault HH. On-line counting of cysteine residues in peptides during electrospray ionization by electrogenerated tags and their application to protein identification. Electrophoresis 2005; 26: 238.

23. Dayon L, Roussel C, Girault HH. Probing cysteine reactivity in proteins by mass spectrometric EC-tagging. J. Proteome Res. 2006; 5: 793.

24. Sechi S, Chait BT. Modification of cysteine residues by alkylation. A tool in peptide mapping and protein identification. Anal. Chem. 1998; 70: 5150.

25. Goodlett DR, Bruce JE, Anderson GA, Rist B, Pasa-Tolic L, Fiehn O, Smith RD, Aebersold R. Protein identification with a single accurate mass of a cysteine-containing peptide and constrained database searching. Anal. Chem. 2000; 72: 1112.

26. Mirzaei H, Regnier F. Structure specific chromatographic selection in targeted proteomics. J. Chromatogr. B Analyt. Technol. Biomed. Life Sci. 2005; 817: 23.

27. Leitner A, Lindner W. Current chemical tagging strategies for proteome analysis by mass spectrometry. J. Chromatogr. B Analyt. Technol. Biomed. Life Sci. 2004; 813: 1.

28. Cech NB, Enke CG. Practical implications of some recent studies in electrospray ionization fundamentals. Mass Spectrom. Rev. 2001; 20: 362.

29. Bonneil E, Tessier S, Carrier A, Thibault P. Multiplex multidimensional nanoLC-MS system for targeted proteomic analyses. Electrophoresis 2005; 26: 4575. 